\title{
Formal Group Laws for Affine Kac-Moody Groups and Group Quantization*
}

\author{
Victor Aldaya ${ }^{1, \star \star}$ and Jose Navarro-Salas ${ }^{2}$ \\ ${ }^{1}$ The Blackett Laboratory, Imperial College, London SW7 2BZ, United Kingdom \\ ${ }^{2}$ Departamento de Fisica Teorica, Facultad de Fisicas, Universidad de Valencia, Burjasot \\ (Valencia), Spain and IFIC (Centro Mixto Universidad de Valencia - C.S.I.C.), Spain
}

\begin{abstract}
We describe a method for obtaining Formal Group Laws from the structure constants of Affine Kac-Moody groups and then apply a group manifold quantization procedure which permits construction of physical representations by using only canonical structures on the group. As an intermediate step we get an explicit expression for two-cocycles on Loop Groups. The programme is applied to the Affine $S U(2)$ group.
\end{abstract}

\section{Introduction}

In recent years Kac-Moody algebras, and the Affine ones in particular, have acquired more and more relevance in Theoretical Physics. Thus, for example, these algebras appear as algebras of hidden symmetries of two-dimensional chiral models [1-3], in self-dual Yang-Mills theory [3], in completely integrable dynamical systems $[4,5]$, Bose-Fermi correspondence $[1,6]$, string theory $[7-9]$, conformally invariant field theories [10], etc.

As is well known [11], there exists an isomorphism between the Affine KacMoody algebra $L(\tilde{A})$ and the unique one-dimensional non-trivial central extension, $C\left[t, t^{-1}\right] \otimes L(A)$, of the gauge-like algebra $C\left[t, t^{-1}\right] \otimes L(A)$. The cocycle $\Sigma$ that fulfills the extension is

$$
\Sigma\left(X \otimes t^{n}, Y \otimes t^{m}\right)=\alpha n \delta_{n,-m} \tau(X, Y), \quad \forall X, Y \in L(A),
$$

where $\tau$ is the killing form of the classical algebra $L(A)$. We have $\operatorname{dim} H^{2}\left(C\left[t, t^{-1}\right] \otimes L(A), C\right)=1$.

\footnotetext{
* Research partially supported by the Conselleria de Cultura de la Generalitat Valenciana, the Plan de Formacion del Personal Investigador, and the Comision Asesora de Investigacion Cientifica y Tecnica (CAICYT)

$\star \star$ On leave of absence from the IFIC, Centro Mixto Universidad de Valencia - C.S.I.C. and the Departamento de Fisica Teorica de la Universidad de Valencia
} 
The above structure for $L(\tilde{A})$ or, rather, the corresponding one for groups, is shared with many basic physical symmetries, the simplest example of which is the central extension of the Galilei group, $\widetilde{G}_{(m)}$, associated with the quantum free particle. This kind of group, and more generally the groups which also have the structure of a principal bundle with $U(1)$ as the structure group (Quantum Groups), has been used to a great extent to obtain the wave functions of physical systems [12-15] through a Group Quantization Formalism (GQF) [12] which only makes use of the group manifold and of the canonical structures defined on it.

In this paper we apply GQF to the principal bundle $\overline{\Omega G}$, the central extension of the space of loops in the simple group $G$, and work out explicitly the wave functions carrying the representations of $\overline{\Omega S U}(2)$ as functions on the group manifold. To start with, the formalism requires a local group law in local coordinates at the identity. We calculate this group law and, in particular, we give an explicit expression for the two-cocycle of the group using the intrinsic group coordinates. In this respect, our procedure diverges from that of reference $[16,17]$. As a general feature the GQF recuperates what would be the Kostant-Souriau Geometric Quantization [18, 19] of the Kirillov-Kostant co-adjoint orbits [19] and solves the problem of the ambiguity of the polarizations in the finite dimensional case as well as that of finding an invariant integration volume. The wave functions that we obtain arise naturally accompanied by a weight function for the invariant scalar product [20].

The fact that we have to resort to a power expansion to find the group law can be seen as a drawback of the procedure. We must note, nevertheless, that the description of a physical system in this way would look like a perturbative one but with the additional advantage of being finite.

We finally remark that obviously GQF provides Hamiltonian (Lagrangian) models in which Affine Kac-Moody algebras are realized not only as infinitesimal actions but also as conserved Noether invariants whose Poisson bracket closes. It must also be noted that "normal ordering" is automatically incorporated into the formalism (through the choice of a polarization) in such a way that central charges already appear at the classical level (in the Hamiltonian-Jacobi version).

\section{II. "Perturbative" Expansion of the Group Law}

Formal Group Theory [21] can be converted into an approximate method for computing local group laws of Kac-Moody groups (although the process can of course be applied to any group). We start with formal power series in two variables of " $Z$ " $\times \operatorname{dim} G+1$ components:

$$
\begin{aligned}
F^{\alpha}\left(X^{\prime}, X\right)= & X^{\prime \alpha}+X^{\alpha}+B_{i, j}^{\alpha} X^{\prime i} X^{j} \\
& +\frac{1}{2 !} B_{i j, \ell}^{\alpha} X^{\prime i} X^{\prime j} X^{\ell}+\frac{1}{2 !} B_{i, j \ell}^{\alpha} X^{\prime i} X^{i} X^{\ell} \\
& +\frac{1}{3 !} B_{i j \ell, m}^{\alpha} X^{\prime i} X^{\prime j} X^{\prime \ell} X^{m} \\
& +\frac{1}{2 !} \frac{1}{2 !} B_{i j, \ell m}^{\alpha} X^{\prime i} X^{\prime j} X^{\ell} X^{m}+\ldots
\end{aligned}
$$


where $i, j, \ell, m \in Z \times\{1,2, \ldots \operatorname{dim} G\}$ and $\alpha=i, j, \ldots$ or $\phi$, the $U(1)$ index. For this formal series to be a formal group law we only have to impose the associativity conditions [22],

$$
F\left(X^{\prime \prime}, F\left(X^{\prime}, X\right)\right)=F\left(F\left(X^{\prime \prime}, X^{\prime}\right), X\right), \quad \forall X^{\prime \prime}, X^{\prime}, X .
$$

The structure constants of the Lie algebra are obtained from the expressions

$$
B_{i, j}^{\alpha}-B_{j, i}^{\alpha}=C_{i j}^{\alpha},
$$

to be referred to in the sequel as the second order conditions.

As is well known [21] the Lie algebra determines the formal group structure and, accordingly, the constants $B_{i_{1} \ldots i_{n}, j_{n+1} \ldots j_{m}}^{\alpha}(m>2)$ are determined modulo a group isomorphism by the structure constants $C_{i j}^{\alpha}$. From condition (2.2) a set of constraint equations follow for $B_{i_{1} \ldots i_{n}, j_{n}+1 \ldots j_{m}}^{\alpha}$ order by order. Thus, for example, the lower orders look like:

$3^{\text {rd }}$ order

$$
B_{i, j \ell}^{\alpha}-B_{i j, \ell}^{\alpha}=B_{p, \ell}^{\alpha} B_{i, j}^{p}-B_{i, p}^{\alpha} B_{j, \ell}^{p},
$$

$4^{\text {th }}$ order

$$
\left[\begin{array}{l}
B_{i, j \ell m}^{\alpha}-B_{i j, \ell m}^{\alpha} \\
\quad=B_{p, \ell m}^{\alpha} B_{i, j}^{p}-B_{i, p}^{\alpha} B_{j, \ell m}^{p}-B_{i, m p}^{\alpha} B_{j, \ell}^{p}-B_{i, \ell p}^{\alpha} B_{j, m}^{p}, \\
B_{i j, \ell m}^{\alpha}-B_{i j \ell, m}^{\alpha} \\
\quad=B_{p, m}^{\alpha} B_{i j, \ell}^{p}+B_{p j, m}^{\alpha} B_{l, \ell}^{p}+B_{p l, m}^{\alpha} B_{j, \ell}^{p}-B_{i j, p}^{\alpha} B_{\ell, m}^{p} .
\end{array}\right.
$$

In general, for $n \geqq 3$ there exist $n-2$ independent equations (corresponding to $a=1,2, \ldots, n-2)$ :

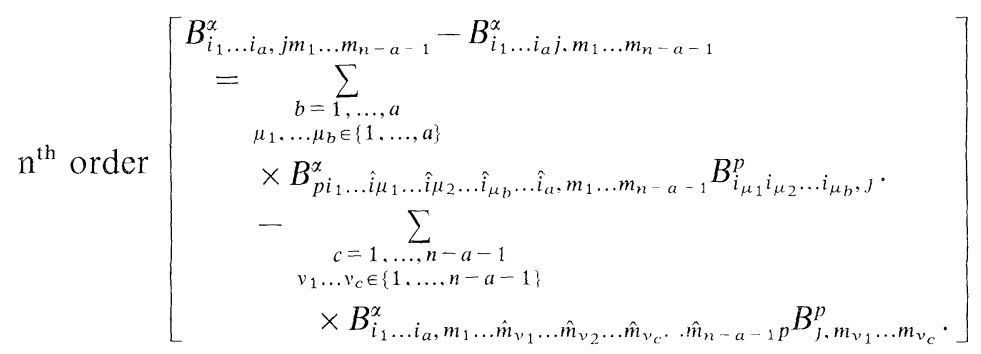

The system (2.6) has the Jacobi identity as the compatibility condition. By successively solving the different sets of equations the formal group law is "perturbatively" derived. It is important to realize that this perturbative expansion is not uniquely determined; there is a certain freedom in the way we choose the $B$ 's in the left-hand side of (2.3)-(2.6) to each order [23]. This "gauge" freedom, which in general corresponds to a group isomorphism, corresponds in particular to the coboundary ambiguity of the central extensions in the case of Affine Kac-Moody groups. Then a rule is needed in working out any formal group law. We point out, however, that GQF is unsensitive to the special choice of the rule so that we may adopt the more advantageous one in each case.

\section{Formal Group Law for $\widetilde{\Omega S U(2)}$}

In the concrete case of the group $\widetilde{\Omega S U}(2)$ the above procedure can be simplified due to the fact that we can write down a formal group law for the $S U(2)$ subgroup. A 
formal Laurent expansion of the parameters of the $S U(2)$ group then provides the solution for all the $B$ 's but for those with upper index $\phi$. We write the local $S U(2)$ group law in a fibre bundle chart of the Hopf fibration $S U(2) \rightarrow S^{2}: \eta=e^{i \theta / 2}$ parametrizes the fibre and $2 z, 2 z^{*}$ the north pole neighbourhood [24]

$$
\begin{aligned}
z^{\prime \prime} & =z^{\prime} \eta^{-2}+x_{3}^{\prime} z-\frac{2 z}{1+x_{3}}\left[z^{*} z^{\prime} \eta^{-2}+z^{*} z \eta^{2}\right], \\
z^{\prime \prime} & =z^{*} \eta^{2}+x_{3}^{\prime} z^{*}-\frac{2 z^{*}}{1+x_{3}}\left[z z^{*} \eta^{2}+z^{\prime} z^{*} \eta^{-2}\right], \\
\eta^{\prime \prime} & =\sqrt{\frac{2}{1+x_{3}^{\prime \prime}}}\left\{\sqrt{\frac{1+x_{3}}{2}} \sqrt{\frac{1+x_{3}^{\prime}}{2}} \eta^{\prime} \eta-\sqrt{\frac{2}{1+x_{3}^{\prime}}} \sqrt{\frac{2}{1+x_{3}}} z^{*} z^{\prime} \eta^{*} \eta^{\prime}\right\}, \\
x_{3} & \equiv \sqrt{1-4 z z^{*}}, \quad x_{3}^{\prime \prime}=x_{3}^{\prime} x_{3}-2\left(z^{*} z^{\prime} \eta^{-2}+z^{* \prime} z \eta^{2}\right) .
\end{aligned}
$$

In these coordinates a formal group law for $\Omega S U(2)$ is given by:

$$
\begin{aligned}
z_{n}^{\prime \prime}= & z_{n}^{\prime}+z_{n}-i z_{n-m}^{\prime} \theta_{m}-z_{n-m-p}^{\prime} z_{m} z_{p}^{*}-z_{n-m-p}^{\prime *} z_{m} z_{p} \\
& -2 z_{n-m-p}^{\prime} z_{m}^{*} z_{p}-\frac{1}{2} z_{n-m-p}^{\prime} \theta_{m} \theta_{p}+\ldots, \\
z_{n}^{\prime * *}= & z_{n}^{\prime *}+z_{n}^{*}+i z_{n-m}^{* *} \theta_{m}-z_{n-m-p}^{* *} z_{m}^{*} z_{p}-z_{n-m-p}^{\prime} z_{m}^{*} z_{p}^{*} \\
& -2 z_{n-m-p}^{\prime *} z_{m}^{\prime} z_{p}^{*}-\frac{1}{2} z_{n-m-p}^{*} \theta_{m} \theta_{p}+\ldots, \\
\theta_{n}^{\prime \prime}= & \theta_{n}^{\prime}+\theta_{n}+i\left(z_{n-m}^{\prime} z_{m}^{*}-z_{n-m}^{\prime *} z_{m}\right)+\left(z_{n-m-p}^{\prime} z_{m}^{*}+z_{n-m-p}^{*} z_{m}\right) \theta_{p}+\ldots,
\end{aligned}
$$

where summation of repeated indices is understood.

The procedure of Sect. II is now applied to extend the group law (3.2) to that of $\widetilde{\Omega S U}(2)$, i.e., to compute explicitly the group two-cocycle $\zeta$. Using the "gauge"

$$
\begin{gathered}
B_{i, j}^{\phi}=-B_{j, i}^{\phi}, \quad i, j=z_{n}, z_{m}^{*}, \theta_{p}, \\
B_{z_{n} z_{m}^{*}, \theta_{p}}^{\phi}=0=B_{\theta_{p}, z_{n} z_{m}^{*}, \quad}^{\phi}, \quad \forall n, m, p \in \mathbb{Z},
\end{gathered}
$$

we obtain for $\phi^{\prime \prime}$,

$$
\begin{gathered}
\phi^{\prime \prime}=\phi^{\prime}+\phi+\alpha \xi\left(g^{\prime}, g\right), \quad g^{\prime}, g \in \Omega S U(2), \\
\xi\left(g^{\prime}, g\right)=\frac{1}{2}\left[n\left(2 z_{n}^{\prime *} z_{-n}+2 z_{n}^{\prime} z_{-n}^{*}+\theta_{n}^{\prime} \theta_{-n}\right)+i(m-n) z_{n}^{\prime} z_{m}^{*} \theta_{n-m}\right. \\
\left.-i(m-n) z_{n}^{\prime *} z_{m} \theta_{-n-m}+i n \theta_{n}^{\prime} z_{m} z_{-n-m}^{*}-i \theta_{n}^{\prime} z_{m}^{\prime *} z_{-n-m}+\ldots\right] .
\end{gathered}
$$

Had we taken another "gauge" for computing the $B^{\phi}$ 's we would have obtained an equivalent (i.e., differing in a two-coboundary) two-cocycle $\hat{\xi}$.

\section{Group Quantization and Representations: The $\overline{\Omega S U}(2)$ case}

The basic structure of the GQF [12] is a Lie group $\widetilde{G}$, the "quantum" group, which in turn is a (say) right $U(1)$-principal bundle (or a $R$-principal bundle then leading to a classical theory) with a 1 -form $\Theta$ naturally selected among the left-invariant 1 -forms which is a connection form. In the simplest case, and roughly speaking, $\widetilde{G}$ 
might be either a (global) central extension of a group $G$ by $U(1)$, characterized by a cocycle $\xi \in H^{2}(G, U(1))$, or a finite dimensional semisimple group with $U(1)$ in the Cartan subgroup. In both cases a vertical left-invariant 1-form is globally defined either by cohomological criteria [20], in the first case, or by means of the Killing form from the fundamental (vertical) left-invariant generator of $\widetilde{G}$ in the second one. However, a semisimple quantum group $\widetilde{G}$ can also be (equivalently) handled as if it were a central extension by making use of a "pseudo-extension" [25], i.e., a trivial extension by $U(1)$ which modifies the structure constants of the algebra. This can only happen if the cocycle, "pseudo-cocycle," is locally generated by a linear function on the parameter of the structure subgroup $[U(1)$ or $R]$ of the principal bundle $\widetilde{G}$. This set of coboundaries is a group strongly related to the cohomology group of a contraction. The connection form $\Theta$ for pseudo-extended groups is obtained as the left-invariant 1 -form dual to the central $U(1)$ generator. We must remark, nevertheless, that in spite of the local considerations just made about the pseudo-cocycle, the associated form is globally defined [see later in this section the comments concerning the $S U(2)$ pseudo-extensions]. This construction was explicitly done in the case of the Poincare group considered as a principal bundle having the time translation subgroup as the structure group [15].

Going from finite to infinite dimension opens new possibilities as the Whitehead lemma no longer holds. Thus, for example, central extensions are not forbidden for semisimple Lie groups.

The Full Polarization $\mathscr{P}$, which generalizes that of ordinary Geometric Quantization $[18,19]$ is defined as a maximal horizontal (with respect to the connection $\Theta$ ) subalgebra of left invariant vector fields containing the characteristic subalgebra of $\Theta[12,15]$, i. e., the algebra generated by the left invariant vector fields that annihilate $\Theta$ and $d \Theta[26]$ :

$$
\mathscr{G}_{\Theta}=\left\langle\tilde{X}^{L} \in \mathcal{X}^{L}(\widetilde{G}) / i_{\tilde{X}^{L}} \Theta=0=i_{\tilde{X}^{L}} d \Theta\right\rangle .
$$

Thus, the full set of conditions on $\Psi \in \mathscr{F}(\widetilde{G}, \mathbb{C})$ characterizing the wave functions are

$$
\Xi \cdot \Psi=i \Psi, \quad \widetilde{X}^{L} \cdot \Psi=O, \quad \forall \tilde{X}^{L} \in \mathscr{P},
$$

where $\Xi$ is the fundamental vector field (generator) of the principal bundle $\widetilde{G}$. On these functions $\Psi$, which are constrained by means of left invariant vector fields, the right invariant vector fields act as ordinary derivations defining the physical operators of the theory. The fact that (in general) left and right vector fields commute is important for the stability of the wave functions subspace under the operator actions.

To apply the GQF to the special case of $\widetilde{\Omega S U}(2)$ we start writing a (formal) group law which exhibits a specific principal bundle structure. To this end we note that besides the principal bundle structure associated with the local central extension characterized by (say) the cocycle $\xi$ in (3.4), the Hopf fibration of the $S U(2)$ subgroup itself defines a $Z$-parametrized family of pseudo-extensions to be taken into account in writing the group law for the $\phi$ parameter. In fact, these pseudo-extensions precisely account for the irreducible representations of the $S U(2)$ subgroup; otherwise $S U(2)$ would be trivially represented. As above mentioned, these pseudo-extensions are realized by means of coboundaries locally generated by linear functions on the local parameter $\theta_{0}$ of the $U(1)$ subgroup of 
$S U(2)[27]\left(\xi_{\mathrm{cob}}\left(g^{\prime}, g\right)=\delta\left(g^{\prime} * g\right)-\delta\left(g^{\prime}\right)-\delta(g)\right)$. Taking into account the global structure of the involved subgroups, the only possible group laws for the $U(1)$ central subgroup of the pseudo-extended group are $\left(\zeta \equiv e^{i \phi}, \eta_{0} \equiv e^{i \theta_{0} / 2}=e^{i \delta(g)}\right)$,

$$
\zeta^{\prime \prime}=\zeta^{\prime} \zeta\left(\eta_{0}^{\prime \prime} \eta_{0}^{\prime-1} \eta_{0}^{-1}\right)^{N}, \quad N \in \mathbb{Z}
$$

Thus, an integer constant (the winding number) appears in the characterization of the representations.

Equation (4.3) along with (3.1) constitute the local group law adapted to the globally defined $U(1)$ pseudo-extended $S U(2)$ group, $S U(2) \widetilde{\otimes} U(1)$. As a principal bundle $S U(2) \widetilde{\otimes} U(1) \rightarrow S U(2)$ is characterized by the Čech-coboundary $\tilde{g}_{\alpha \beta}(\xi)$ $=\pi^{*} g_{\alpha \beta}(\xi) \equiv g_{\alpha \beta}\left(\xi^{+} \sigma \xi\right)$, where $\pi$ is the $S U(2) \rightarrow S^{2}$ projection, $g_{\alpha \beta}$ is a Čech-cocycle associated with the $\mathrm{SU}(2) \rightarrow \mathrm{S}^{2}$ fibration, $\xi \in S^{3} \subset C^{2}$ and $\sigma$ are the Pauli matrices.

We now propose as the final group law for $\widetilde{\Omega S U}(2)$ the law given by (3.2) plus

$$
\begin{gathered}
\phi^{\prime \prime}=\phi^{\prime}+\phi+\alpha \xi\left(g^{\prime}, g\right)+N \xi_{\mathrm{cob}}\left(g^{\prime}, g\right), \\
\xi_{\mathrm{cob}}\left(g^{\prime}, g\right)=-i \ln \left(\eta_{0}^{\prime \prime} \eta_{0}^{\prime-1} \eta_{0}^{-1}\right) \\
=\frac{1}{2}\left\{i\left(z_{-m}^{\prime} z_{m}^{*}-z_{-m}^{\prime *} z_{m}\right)+\left(z_{-m-p}^{\prime} z_{m}^{*}+z_{-m-p}^{*} z_{m}\right) \theta_{p}+\ldots\right\} .
\end{gathered}
$$

From the group law (3.2), (4.4) we only have to derive the left-invariant fields, the $\Theta$ form (dual to the $\phi$ generator) by means of the duality relations and to obtain the Full Polarization subalgebra. We shall proceed, of course, order by order.

Left-Invariant Vector Fields:

$$
\begin{aligned}
\tilde{X}_{z_{q}}^{L}= & \frac{\partial}{\partial z_{q}}-i z_{n-q}^{*} \frac{\partial}{\partial \theta_{n}}-2 z_{m} z_{n-m-q}^{*} \frac{\partial}{\partial z_{n}} \\
& +\ldots-\left[\left(\alpha q+i \frac{N}{2}\right) z_{-q}^{*}+\frac{i \alpha}{2} n \theta_{n} z_{-n-q}^{*}+\ldots\right] \Xi, \\
\tilde{X}_{z_{q}}^{L^{*}=} & \frac{\partial}{\partial z_{q}^{*}}+i z_{n-q} \frac{\partial}{\partial \theta_{n}}-2 z_{m}^{*} z_{n-m-q} \frac{\partial}{\partial z_{n}^{*}} \\
& +\ldots-\left[\left(\alpha q-i \frac{N}{2}\right) z_{-q}-i \frac{\alpha}{2} n \theta_{n} z_{-n-q}+\ldots\right] \Xi, \\
\tilde{X}_{\theta_{q}}^{L}= & \frac{\partial}{\partial \theta_{q}}-i z_{n-q} \frac{\partial}{\partial z_{n}}+i z_{n-q}^{*} \frac{\partial}{\partial z_{n}^{*}}+\left[-\frac{\alpha}{2} q \theta_{q}+\ldots\right] \Xi, \\
\tilde{X}_{\phi}^{L} \equiv & \Xi=\frac{\partial}{\partial \phi} .
\end{aligned}
$$

Lie Algebra Commutators:

$$
\begin{aligned}
& {\left[\tilde{X}_{z_{q}}^{L}, \tilde{X}_{z_{p}^{*}}^{L}\right]=2 i \tilde{X}_{\theta_{q+p}}^{L}+2\left(\alpha q+i \frac{N}{2}\right) \delta_{q,-p} \Xi,} \\
& {\left[\tilde{X}_{\theta_{q}}^{L}, \tilde{X}_{z_{p}}^{L}\right]=i \tilde{X}_{z_{q+p}}^{L}, \quad\left[\tilde{X}_{\theta_{q}}^{L}, \tilde{X}_{z_{p}^{*}}^{L}\right]=-i \tilde{X}_{z_{q}^{*}+p}^{L},} \\
& {\left[\tilde{X}_{\theta_{q}}^{L}, \tilde{X}_{\theta_{p}}^{L}\right]=\alpha q \delta_{q,-p} \Xi .}
\end{aligned}
$$


Quantization Form $\Theta$ :

$$
\begin{aligned}
\Theta= & \left(\alpha q+i \frac{N}{2}\right)\left[z_{-q}^{*} d z_{q}-z_{q} d z_{q}^{*}\right]+\frac{\alpha}{2} q \theta_{-q} d \theta_{q} \\
& +i\left[\left(\alpha n+i \frac{N}{2}\right) z_{n-q^{*}} z_{-n}^{*}+\left(-\alpha n+i \frac{N}{2}\right) z_{n-q_{-n}^{*}}^{*}\right] d \theta_{q}+\ldots+d \phi .
\end{aligned}
$$

Characteristic Subalgebra. Both solving the equations $i_{\tilde{X}} \Theta=0=i_{\tilde{X}} d \Theta$, we find the following possibilities (depending on the values of $\alpha$ and $N$ ):

$$
\mathscr{G}_{\Theta} \text { generated by }\left[\begin{array}{lll}
\tilde{X}_{\theta_{0}}^{L} & \text { for } & \forall \alpha \neq 0, N \neq 0 \\
\tilde{X}_{\theta_{0}}^{L}, \tilde{X}_{z_{0}}^{L}, \tilde{X}_{z_{0}^{*}}^{L^{*}} & \text { for } & \forall \alpha \neq 0, N=0 .
\end{array}\right.
$$

Equation (4.8b) corresponds to a $S U(2)$ subalgebra [indeed (4.8b) is the original $S U(2)$ subalgebra] while (4.8a) can be seen as the Cartan subalgebra of $S U(2)$.

Full Polarization Subalgebra. Unlike the finite dimensional case there may exist more than one non-equivalent polarization. Indeed we are faced with a "no conjugacy" theorem similar to that of Borel subalgebras [28] of infinite dimensional semisimple algebras [29] to which polarizations parallel. This means that non-equivalent dynamics are associated with different unequivalent polarizations. To each one of the possible choices of $\mathscr{G}_{\Theta}$ we find the following essentially different polarizations (any other is obtained by conjugacy):

$$
\begin{gathered}
\mathscr{G}_{\theta}=\left\langle\tilde{X}_{\theta_{0}}^{L}\right\rangle \rightarrow\left\{\begin{array}{l}
\mathscr{P}_{a}=\left\langle\tilde{X}_{\theta_{n} \leqq 0}^{L}, \tilde{X}_{z_{q}}^{L}\right\rangle \\
\mathscr{P}_{a}^{\prime}=\left\langle\tilde{X}_{\theta_{n} \leqq 0}^{L}, \tilde{X}_{z_{q \leqq 0}}^{L}, \tilde{X}_{z_{q}^{*}<0}^{L}\right\rangle,
\end{array}\right. \\
\mathscr{G}_{\theta}=\left\langle\tilde{X}_{\theta_{0}}^{L}, \tilde{X}_{z_{0}}^{L}, \tilde{X}_{z_{0}^{*}}^{L_{0}}\right\rangle \rightarrow \mathscr{P}_{b}=\left\langle\tilde{X}_{\theta_{n} \leqq 0}^{L}, \tilde{X}_{z_{q} \leqq 0}^{L}, \tilde{X}_{z_{q}^{*} \leqq 0}^{L}\right\rangle,
\end{gathered}
$$

Mimicking the denomination used for Borel subalgebras [29] we call (4.9a) the natural polarization while $\left(4.9 \mathrm{a}^{\prime}\right)$ will be referred to as the standard one. It must be noted that $\left(4.9 a^{\prime}\right)$ and $(4.9 b)$ have the same structure; $(4.9 b)$ is obtained from $\left(4.9 \mathrm{a}^{\prime}\right)$ by adding to it the generator $\tilde{X}_{z_{0}^{*}}^{L_{*}}$ which appears in the corresponding $\mathscr{G}_{\Theta}$.

We shall be concerned in what follows with the more general case of $(4.8 \mathrm{a})$ and, in turn, restricted to the (4.9a) choice which is going to provide us with a set of non-explicitly worked out (Verma module) representations. Exactly the same procedure can be applied in the other possibilities. It will be done elsewhere.

Wave Functions. The wave functions are the $U(1)$-functions on $\overline{\Omega S U}(2)$ satisfying the Full Polarization conditions, i.e., functions on $\widetilde{\Omega S U}(2)$ verifying $\Xi \cdot \Psi=i \Psi$ and

$$
\tilde{X}_{\theta_{n}}^{L} \cdot \Psi=0, \quad \tilde{X}_{z_{q}}^{L} \cdot \Psi=0, \quad n, q \in \mathbb{Z}, n \leqq 0 .
$$


Equations (4.10) can be solved order by order leading to the following set of "Fock" states:

$$
\begin{aligned}
& |\alpha, N\rangle \sim \zeta \cdot W\left(\theta, z, z^{*} ; \alpha, N\right), \quad \text { (the vacuum) } \\
& \left|\alpha, N ; \theta_{n}\right\rangle \sim \zeta \cdot W \cdot\left\{\theta_{n}+i \sum_{p} z_{n-p} z_{p}^{*}+O(4)\right\}, \quad n>0, \\
& \left|\alpha, N ; \theta_{n} \theta_{m}\right\rangle \sim \zeta \cdot W \cdot\left\{\theta_{n}+i \sum_{p} z_{n-p} z_{p}^{*}+\ldots\right\} \\
& \quad \times\left\{\theta_{m}+i \sum_{p} z_{m-p} z_{p}^{*}+\ldots\right\}, \quad n, m>0,
\end{aligned}
$$

$$
\begin{aligned}
\left|\alpha, N ; z_{q}^{*}\right\rangle \sim \zeta & \cdot W \cdot e^{-i \theta_{0}} \times\left\{z_{q}^{*}-i \sum_{q-m<0} \theta_{q-m_{m}} z_{m}^{*}-\frac{1}{2} \sum_{\substack{q-p-m<0 \\
p<0}} \theta_{q-p-m} \theta_{p} z_{m}^{*}\right. \\
+ & \left.\sum_{q-p \leqq 0} z_{q-p-m} z_{p}^{*} z_{m}^{*}+\ldots\right\}, \quad q \in \mathbb{Z}, \\
\left|\alpha, N ; z_{q}^{*} z_{k}^{*}\right\rangle \sim & \cdot W \cdot e^{-2 i \theta_{0}} \\
& \times\left\{z_{q}^{*}-i \sum_{q-m<0} \theta_{q-m_{m}} z_{m}^{*}-\frac{1}{2} \sum_{q-p-m<0} \cdots\right\} \\
& \times\left\{z_{k}^{*}-i \sum_{k-m<0} \theta_{k-m} z_{m}^{*}-\frac{1}{2} \underset{k-p-m<0}{\sum_{k<0} \cdots}\right\}, \quad q, k \in \mathbb{Z},
\end{aligned}
$$

$$
\begin{aligned}
\left|\alpha, N ; \theta_{n} z_{q}^{*}\right\rangle \sim & \zeta \cdot W \cdot e^{-i \theta_{0}}\left\{\theta_{n}+i \sum_{p} z_{n-p} z_{p}^{*}+\ldots\right\} \\
& \times\left\{z_{q}^{*}-i \sum_{q-m<0} \theta_{q-m} z_{m}^{*}+\ldots\right\}, \quad n>0, q \in \mathbb{Z},
\end{aligned}
$$

where the weight function takes the form

$$
\begin{aligned}
W\left(\theta, z, z^{*} ; \alpha, N\right)= & 1-\frac{N}{2} \sum_{k} z_{k} z_{-k}^{*}+i \frac{\alpha}{2} \sum_{m<0} m \theta_{m} \theta_{-m} \\
& +i \alpha \sum_{m} m z_{m} z_{m}^{*}-\alpha \sum_{m<0} m \theta_{m} z_{p^{2}} z_{-m-p}^{*}+\ldots
\end{aligned}
$$

Once the wave functions are known, the group law [(3.2), (4.4)] can be used to obtain group representations parametrized by $\alpha$ and $N$. This is accomplished by means of the left translations acting on $\Psi$,

$$
L_{\tilde{g}^{\prime}} \Psi(\tilde{g})=\Psi\left(\tilde{g}^{\prime} * \tilde{g}\right), \quad \tilde{g}^{\prime}, \tilde{g} \in \widetilde{\Omega S U}(2) .
$$


As mentioned earlier the right-invariant vector fields $\tilde{X}^{R}$ (which generate the finite left translations) act on the wave functions as ordinary derivations and define the basic (Lie algebra) operators.

Right-Invariant Vector Fields: Basic Operators and Lie Algebra Representations:

$$
\begin{aligned}
& \tilde{X}_{z_{q}}^{R}= \frac{\partial}{\partial z_{q}}-i \theta_{n-q} \frac{\partial}{\partial z_{n}}+i z_{n-q}^{*} \frac{\partial}{\partial \theta_{n}} \\
&-z_{m} z_{n-m-q}^{*} \frac{\partial}{\partial z_{n}}-\frac{1}{2} \theta_{m} \theta_{n-m-q} \frac{\partial}{\partial z_{n}} \\
&-z_{m}^{*} z_{n-m-q}^{*} \frac{\partial}{\partial z_{n}^{*}}+z_{m}^{*} \theta_{n-m-q} \frac{\partial}{\partial \theta_{n}}+\ldots+\left[\left(\alpha q+i \frac{N}{2}\right) z_{-q}^{*}\right. \\
&\left.+\left(i \frac{\alpha}{2}(n-q)+\frac{N}{2}\right) z_{n}^{*} \theta_{-n-q}+\ldots\right] \Xi, \\
& \frac{\partial}{\partial z_{q}^{*}}+i \theta_{n-q} \frac{\partial}{\partial z_{n}^{*}}-i z_{n-q} \frac{\partial}{\partial \theta_{n}}-z_{m}^{*} z_{n-m-q} \frac{\partial}{\partial z_{n}^{*}}-\frac{1}{2} \theta_{m} \theta_{n-m-q} \frac{\partial}{\partial z_{n}^{*}} \\
&-z_{m} z_{n-m-q} \frac{\partial}{\partial z_{n}}+z_{m} \theta_{n-m-q} \frac{\partial}{\partial \theta_{n}}+\ldots+\left[\left(\alpha q-i \frac{N}{2}\right) z_{-q}\right. \\
&\left.+\left(-i \frac{\alpha}{2}(n-q)+\frac{N}{2}\right) z_{n} \theta_{-n-q}+\ldots\right] \Xi, \\
& \tilde{X}_{\theta_{q}}^{R}=\frac{\partial}{\partial \theta_{q}}+\left[\frac{\alpha}{2} q \theta_{-q}+\ldots\right] \Xi .
\end{aligned}
$$

The derivative of the wave functions with respect to (4.14) provide an approximate expression for the basic operators (indeed a $3^{\text {rd }}$ order one) that, of course, could be improved by going further in the group law series. Nevertheless this $3^{\text {rd }}$ order calculation allows us to conjecture a full expansion which is a posteriori tested by making use of the Lie algebra commutators and the fact that part of the operators are exactly known. As an example we give the action of $\tilde{X}^{R}$ on the basic states, i.e., $[\alpha, N\rangle,\left[\alpha, N ; \theta_{n}\right\rangle, n>0$, and $\left[\alpha, N ; z_{q}^{*}\right\rangle, \forall q \in Z$ :

$$
\begin{gathered}
\tilde{X}_{\theta_{q}}^{R}|\alpha, N\rangle=+i \alpha q\left|\alpha, N ; \theta_{-q}\right\rangle, \quad q \leqq 0, \\
\tilde{X}_{\theta_{q}}^{R}|\alpha, N\rangle=0, \quad q>0, \\
\tilde{X}_{\theta_{q}}^{R}\left|\alpha, N ; \theta_{n}\right\rangle=+i \alpha q\left|\alpha, N ; \theta_{n} \theta_{-q}\right\rangle, \quad q \leqq 0, \\
\tilde{X}_{\theta_{q}}^{R}\left|\alpha, N ; \theta_{n}\right\rangle=\delta_{q, n}|\alpha, N\rangle, \quad q>0, \\
\tilde{X}_{\theta_{q}}^{R}\left|\alpha, N ; z_{n}^{*}\right\rangle=-i\left|\alpha, N ; z_{n-q}^{*}\right\rangle+i \alpha q\left|\alpha, N ; z_{n}^{*} \theta_{-q}\right\rangle, \quad q \leqq 0, \\
\tilde{X}_{\theta_{q}}^{R}\left|\alpha, N ; z_{n}^{*}\right\rangle=0, \quad q>0, \\
\left.\tilde{X}_{z_{q}}^{R}|\alpha, N\rangle=-2 \sum_{s} \frac{(-i)^{s}}{s !} \sum_{m_{1} \ldots m_{s}>0}\right] \\
\times\left[\frac{N}{2}-i \alpha\left(q+m_{1}+\ldots+m_{s}\right)\right]\left|\alpha, N ; \theta_{m_{1}} \ldots \theta_{m_{s}} z_{-q-m_{1} \ldots-m_{s}}^{*}\right\rangle,
\end{gathered}
$$




$$
\begin{aligned}
& \tilde{X}_{z_{q}}^{R}\left|\alpha, N ; z_{n}^{*}\right\rangle=-2 \sum_{s} \frac{(-i)^{s}}{s !} \sum_{m_{1} \ldots m_{s}>0} \\
& \times\left[\frac{N}{2}-i \alpha\left(q+m_{1}+\ldots+m_{s}\right)\right]\left|\alpha, N ; \theta_{m_{1}} \ldots \theta_{m_{s}} z_{n}^{*} z_{-q-m_{1} \ldots m_{s}}\right\rangle \\
& +\sum_{p} \operatorname{sgn}(n-p) \sum_{s} \frac{(-i)^{s}}{s !} \sum_{m_{1} \ldots m_{s}>0} \\
& \times\left|\alpha, N ; \theta_{m 1} \ldots \theta_{m_{s}} z_{p}^{*} z_{n-p-q-m_{1} \ldots-m_{s}}^{*}\right\rangle, \\
& \tilde{X}_{z_{q}}^{R}\left|\alpha, N ; \theta_{n}\right\rangle=-2 \sum_{s} \frac{(-i)^{s}}{s !} \sum_{m_{1} \ldots m_{s}>0} \\
& \times\left[\frac{N}{2}-i \alpha\left(q+m_{1}+\ldots m_{s}\right)\right]\left|\alpha, N ; \theta_{n} \theta_{m_{1}} \ldots \theta_{m_{s}} z_{-q-m_{1} \ldots-m_{s}}^{*}\right\rangle \\
& +2 i \sum_{s} \frac{(-i)^{s}}{s !} \sum_{m_{1} \ldots m_{s}>0} \\
& \times\left|\alpha, N ; \theta_{m_{1}} \ldots \theta_{m_{s}} z_{n-q-m_{1}-\ldots-m_{s}}^{*}\right\rangle, \\
& \tilde{X}_{z_{q}^{*}}^{R}|\alpha, N\rangle=0, \\
& \tilde{X}_{z_{q}^{*}}^{R}\left|\alpha, N ; z_{n}^{*}\right\rangle=\delta_{n . q}|\alpha, N\rangle
\end{aligned}
$$

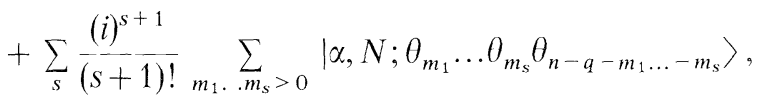

$$
\begin{aligned}
& \tilde{X}_{z_{q}^{*}}^{R}\left|\alpha, N ; \theta_{n}\right\rangle=0 .
\end{aligned}
$$

The expressions for general states $\left[\alpha, N ; \theta \ldots \theta z^{*} \ldots z^{*}>\right.$ are easily obtained because of their factor structure.

Equations (4.15) generalize the much simpler case of the $S U(2)$ representations on Fock states in terms of which coherent ones can be given. Putting $\alpha=0$ in the previous equations, the set of operators $\left(\tilde{X}_{\theta_{0}}^{R}-\frac{N}{2} \tilde{X}_{\zeta}^{R}, \tilde{X}_{z_{q}}^{R}, \tilde{X}_{z^{*} q}^{R}\right)$ for fixed $q \geqq 0$ represents the $S U(2)$ Lie algebra on the subspace $H=\left\{[N\rangle,\left[N ; z_{-q}^{*}\right\rangle\right.$, $\left[N ; z_{-q}^{*} z_{-q}^{*}\right\rangle, \ldots,\left[N ;\left(z_{-q}^{*}\right)^{m}>\ldots\right\}$ :

$$
\begin{aligned}
\left.\tilde{X}_{\theta_{0}}^{R}\right|_{H}\left|N ;\left(z_{-q}^{*}\right)^{m}\right\rangle & =-i\left(m-\frac{N}{2}\right)\left|N ;\left(z_{-q}^{*}\right)^{m}\right\rangle, \\
\left.\tilde{X}_{z_{q}}^{R}\right|_{H}\left|N ;\left(z_{-q}^{*}\right)^{m}\right\rangle & =(m-N)\left|N ;\left(z_{-q}^{*}\right)^{m+1}\right\rangle, \\
\left.\tilde{X}_{z^{*}}^{R}\right|_{q}\left|N ;\left(z_{-q}^{*}\right)^{m}\right\rangle & =m\left|N ;\left(z_{-q}^{*}\right)^{m-1}\right\rangle, \quad m=0, \ldots, N,
\end{aligned}
$$

where $\left[N ; z_{-q}^{*}\right\rangle$ can be now summed up (by ignoring the $\theta_{n}, n \neq 0$, and $z_{p}, z_{-p}^{*}$, $p \neq q)$ :

$$
\begin{aligned}
\left|N ;\left(z_{-q}^{*}\right)^{m}\right\rangle & \sim(1+\chi)^{N / 2}\left[e^{-i \theta_{0}}(1+\chi)^{-1} z_{-q}^{*}\right]^{m}, \\
x & \equiv \sqrt{1-4 z_{-q}^{*} z_{q}} .
\end{aligned}
$$

Equations (4.16) clearly reveal the "spin" character of the half-integer $N / 2$. 
The above operators (4.15) leave invariant the volume $\left(L_{\tilde{X}^{R}} \mu=0\right)$

$$
\mu=\prod_{n, k} \theta_{\left(\theta_{n}\right)}^{L} \wedge \theta_{(\theta-n)}^{L} \wedge \theta_{\left(z_{k}\right)}^{L} \wedge \theta_{\left(z_{-k}^{*}\right)}^{L}, \quad n, k \in Z, n>0,
$$

where $\theta_{(\text {) }}^{L}$ are (horizontal) components of the canonical 1-form, in terms of which an invariant hermitian form could be defined.

We shall discuss in detail these representations as well as those associated with the case (4.8b) in a future publication where the $\overline{\Omega S U}(2)$ group will be enlarged to incorporate the $1+1$ "space-time translations." Here we shall only point out that physical models can be built immediately in this group quantization framework which allows us to construct Noether invariants $\left(i_{\tilde{X}^{R}} \Theta\right)$, equations of motions, etc. $[20,30]$. For instance, for the "Hamiltonian" $-i_{\tilde{X}_{\theta_{0}}^{R}} \Theta$ we obtain

$$
\sum_{n}-i \alpha n z_{-n}^{*} z_{n}+\ldots+\frac{N}{2} \sum_{n} z_{-{ }_{n}{ }_{n}} z_{1}+\ldots,
$$

where a term depending on the winding number $N$ does appear.

The meaning of the characteristic algebra $\mathscr{G}_{\Theta}$ is also worth mentioning. Apart from providing the equations of motions, the characteristic group (the generators of which generate $\left.\mathscr{G}_{\Theta}\right)$ constitutes the symmetry of the vacuum $([\alpha, N\rangle$ in our case $)$, as can be easily derived from the general formalism $[12,20,30]$. In this way the addition to the group law of $\widetilde{\Omega S U}(2)$ of that we could call a "Wess-Zumino" cocycle (pseudo-cocycle indeed) break the original symmetry (4.8b) to (4.8a).

To conclude, we mention that the above procedure can be applied to any Affine Kac-Moody group $\widetilde{\Omega G}$ by following the same steps given here for the $S U(2)$ case. The only peculiarity (apart from the difficulty of writing down the group law for an arbitrary simple group) is that in general the dimension of the pseudo-cohomology group is greater than one. Thus, several indices of the $N$ type must appear. More concretely, for a general finite dimensional simple Lie group $G$ with Cartan matrix $A_{i j}(\operatorname{det} A \neq 0)$, we shall have the following basic commutation relations:

$$
\begin{aligned}
{\left[t^{n} \otimes E_{\beta}, t^{-n} \otimes E_{-\beta}\right] } & =H_{\beta}+\frac{2 n x}{(\beta, \beta)} \Xi+N_{\beta} \delta^{n, 0} \Xi, \\
{\left[t^{n} \otimes H_{i}, t^{-n} \otimes H_{j}\right] } & =\frac{2 n \varkappa A_{i j}}{\left(\beta_{i}, \beta_{i}\right)} \Xi .
\end{aligned}
$$

A simple look at (4.20) permits us to write the first terms in the expansion of the presymplectic form $d \Theta$ :

$$
\begin{aligned}
d \Theta \sim & \frac{2 n \varkappa A_{i j}}{\left(\beta_{i}, \beta_{i}\right)} d h_{i}^{n} \wedge d h_{j}^{-n}+\frac{2 n \varkappa}{(\beta, \beta)} d e_{\beta}^{n} \wedge d e_{-\beta}^{-n}+\ldots \\
& +N_{\beta} \delta^{n, 0} d e_{\beta}^{n} \wedge d e_{-\beta}^{-n}+\ldots,
\end{aligned}
$$

where $h_{i}^{n}$ stand for the parameters associated with the generators $t^{n} \otimes H_{i}$ and $e_{\beta}^{n}$ for those associated with $t^{n} \otimes E_{\beta}$; there are only $l($ rank $\mathscr{G})$ independent winding numbers $N_{\beta}$ and they characterize a highest weight of the Lie algebra of $G, \mathscr{G}$. We 
are able from (4.20) to determine the characteristic subalgebra for the case analogous to that of $(4.8 \mathrm{a})$,

$$
\mathscr{G}_{\Theta}=\langle H\rangle,
$$

and the essentially two unequivalent Full Polarizations,

$$
\begin{gathered}
\mathscr{P}=\left\langle H^{n}, n \leqq 0 ; L_{\beta}^{n}, n \in \mathbb{Z}, \beta>0\right\rangle, \\
\mathscr{P}^{\prime}=\left\langle H^{n}, n \leqq 0 ; L_{\beta}^{n \leqq 0}, L_{-\beta}^{n<0}, \beta>0\right\rangle ;
\end{gathered}
$$

any other Full Polarization can be obtained by restricting some Lie algebra isomorphism to either (4.23) or (4.24). It must be stressed that in general only the class of $\mathscr{P}^{\prime}$ is suitable for unitary representations.

Acknowledgements. We acknowledge the Imperial College for its hospitally. One of us (V.A.) wants to thank D. I. Olive for useful comments on the structure of the Wess-Zumino term in Lagrangian field theory. Both authors thank V. G. Kac for valuable discussions about representation theory of Kac-Moody algebras.

\section{References}

1. Witten, E.: Comm. Math. Phys. 92, 455 (1984)

2. Knizhnik, V.G., Zamolodchikov, A.B.: Nucl. Phys. B 247, 83 (1984)

3. Dolan, L.: Phys. Rep. 109, (1), 1 (1984)

4. Jimbo, M., Miwa, T.: Publ. RIMS, Kyoto Univ. 19, 943 (1983)

5. Olive, D., Turok, N.: Nucl. Phys. B 253, 277 (1985)

6. Mickelsson, J.: Phys. Rev. D 32, 436 (1985)

7. Goddard, P., Olive, D.: Int. J. Mod. Phys. A 1 (2), (1986)

8. Green, M., Schwarz, J.: Phys. Lett. B 149, 117 (1984)

9. Gross, D.J., Harvey, J., E. Martinec, E., Rohm, R.: Nucl. Phys. B 256, 253 (1985)

10. Schellenkens, A.N.: CERN TH 4405/86

11. Kac, V.G.: Infinite dimensional Lie algebras. Boston: Birkhäuser 1983

12. Aldaya, V., de Azcarraga, J.A.: J. Math. Phys. 23, 1297 (1982)

13. Aldaya, V., de Azcarraga, J.A., Wolf, K.B.: J. Math. Phys. 25, 506 (1984)

14. Aldaya, V., de Azcarraga, J.A.: Phys. Lett. B 121, 331 (1983)

15. Aldaya, V., de Azcarraga, J.A.: Ann. Phys. (NY) 165, 484 (1985)

16. Mickelsson, J.: Phys. Rev. Lett. 55, 2099 (1985)

17. Pressley, A., Segal, G.: Loop groups. Oxford University Press 1986

18. Souriau, J.M.: Structure des systemes dynamiques. Paris: Dunod 1970

19. Kostant, B.: Quantization and unitary representations. In: Lecture Notes in Mathematics, Vol. 170. Berlin, Heidelberg, New York: Springer 1970

20. Aldaya, V., de Azcarraga, J.A.: Fortschr. Phys. 35 (6), 437 (1987)

21. Serre, J.P.: Lie algebras and Lie groups. New York: Benjamin 1965

22. The form of the first order terms guarantees that the further conditions $F\left(X^{\prime}, 0\right)=0=F(0, X)$ are always satisfied. It must be realized that our co-ordinates in (2.1) do not have to be those of a canonical system at the identity (i.e., $u^{i}(g) / g=e^{u^{\imath}(g) X_{(1)}}$, where $g \in G,\left\{X_{(l)}\right\}$ is a basis of the Lie algebra)

23. One possible solution is the Baker-Campbell-Hausdorf formula. However this formula has the drawback that the principal bundle structure of a simple Lie group is not manifest in the local group law 
24. Aldaya, V., de Azcarraga, J.A.: In: Modern developments in analytical mechanics. Acta Acad. Sci. Taurinensis 117, [Suppl.] 1 (1983) [Benenti, S., Francaviglia, M., Lichnerovicz, A. (eds.) Torino]

25. The "pseudo-cohomology" also arise in the physical realizations of the Virasoro algebra through the term $-m \delta_{m,-n}$ in the right-hand side of the commutator $\left[L_{m}, L_{n}\right]$

26. When the dimension of the group is finite, the inclusion of $\mathscr{G}_{\Theta}$ in $\mathscr{P}$ makes the procedure uniquely defined (up to an isomorphism of the theory)

27. Much in the same way the pseudo-extensions of the Poincare group are generated by the function $m c x_{0}$, where $m \in R$ is the mass, $c$ the velocity of light and $x_{0}$ parametrizes the time translations [15]

28. Humphreys, J.E.: Introduction to Lie algebras and representation theory. Graduate Texts in Mathematics, Vol. 9. Berlin, Heidelberg, New York: Springer 1972

29. Jacobsen, H.P., Kac, V.: A new class of unitarizable highest weight representations of infinite dimensional Lie algebras. In: Non-linear equations in classical and quantum field theory. Sanchez, N. (ed.) pp. 1-20. Lecture Notes in Physics, Vol. 226. Berlin, Heidelberg, New York: Springer 1985

30. Aldaya, V., de Azcarraga, J.A.: Proceedings of the Conference on "Constraint's theory and relativistic dynamics". Firence 1986 World Scientific Publ. (G. Longhi and L. Lusana eds.) (1987)

31. The classification of the different special coboundaries was made in terms of the winding number of applications from the structure group of the $S U(2) \rightarrow S^{2}$ fibration into the $U(1)$ central subgroup of $\widetilde{\Omega S U}(2)$

Communicated by A. Jaffe

Received February 10, 1987; in revised form May 13, 1987 
